\title{
Il saute dehors/ Il grimpe dessus : particules adverbales de trajectoire en français L1 et L2
}

Il saute dehors/ Il grimpe dessus: The Marking of Trajectory with Adverbial Particles in L1 and L2 French

Marie-Eve Michot, Stefanie Goldschmitt et Michel Pierrard

\section{OpenEdition}

\section{Journals}

Édition électronique

URL : http://journals.openedition.org/pratiques/2773

DOI : $10.4000 /$ pratiques. 2773

ISSN : 2425-2042

Éditeur

Centre de recherche sur les médiations (CREM)

Référence électronique

Marie-Eve Michot, Stefanie Goldschmitt et Michel Pierrard, « I/ saute dehors/ II grimpe dessus :

particules adverbales de trajectoire en français L1 et L2 », Pratiques [En ligne], 167-168| 2015, mis en ligne le 01 avril 2016, consulté le 03 mai 2019. URL : http://journals.openedition.org/pratiques/2773 ; DOI : 10.4000/pratiques.2773

Ce document a été généré automatiquement le 3 mai 2019.

(c) Tous droits réservés 


\section{Il saute dehors/ Il grimpe dessus : particules adverbales de trajectoire en français L1 et L2}

Il saute dehors/ Il grimpe dessus: The Marking of Trajectory with Adverbial Particles in L1 and L2 French

Marie-Eve Michot, Stefanie Goldschmitt et Michel Pierrard

1 Les études typologiques sur l'expression du mouvement (Talmy, 1985, 2000 ; Berman \& Slobin, 1994) ont souligné une différence radicale entre les langues romanes et germaniques dans la réalisation linguistique de tels énoncés. Ces langues s'opposent en particulier par la manière dont elles expriment la trajectoire (marquage de la direction ou de la position) au moyen de syntagmes prépositionnels et de particules adverbales.

2 Notre contribution est une première étude exploratoire visant à évaluer l'impact du contraste invoqué sur la production par des germanophones en français L2 de particules adverbales « exclues » en français L1.

\section{Cadre général}

\subsection{L'expression du mouvement dans les langues romanes et germaniques}

3 Le mouvement représente un concept universel réalisé sous diverses matrices de lexicalisation dans les langues spécifiques. Selon les études examinant les évènements exprimant le mouvement (e.g. Talmy, 1985, 2000 ; Berman \& Slobin 1994 ; Slobin 1996, 2004, 2006 ; Hickmann, 2003), les langues romanes et germaniques utilisent des stratégies différentes pour exprimer ceux-ci :

- les langues romanes préfèrent en général les verbes de mouvement qui marquent une direction ou une trajectoire («path»; p.ex. en français sortir, descendre, monter ou en espagnol salir, bajar, montar). En revanche, les langues germaniques favorisent les verbes de 
mouvement qui expriment la manière de bouger («manner»; p. ex. en anglais run, roll, creep, en néerlandais rennen, lopen, springen ou en allemand rennen, eilen, schleichen);

- de plus, les premières sont des V-langues (centrées sur le verbe) dans la mesure où c'est celui-ci qui exprime l'orientation du mouvement. Les secondes par contre sont des S-langues (centrées sur le satellite) dans le sens où la trajectoire y est souvent indiquée par une particule adverbale (donc ajoutée aux verbes) comme en anglais in, out, up, ou en néerlandais in, uit, buiten, binnen, boven ou en allemand hinaus, hinein, hinauf:

(1a) anglais : The boy ran out (of the house).

(1b) néerlandais : De jongen loopt het huis uit.

(1c) allemand : Der Junge rannte (aus dem Haus) hinaus.

(1d) français : Le garçon sortait (de la maison) en courant.

(1e) espagnol : El chico salió (de la casa) corriendo.

\subsection{L'impact de la $\mathrm{L} 1$ sur la production de la $\mathrm{L} 2$}

L'impact de l'agencement linguistique de la L1 sur la production en L2 est controversé en acquisition. Néanmoins, des études récentes ( $c f$. e.g. De Lorenzo, 2002; Demagny \& Paprocka, 2004) montrent que la structure conceptuelle du système linguistique de la L1 exerce son influence sur la conceptualisation du message verbal exprimé en langue seconde, y compris dans la production d'apprenants au stade ultime de l'acquisition de la L2. M. Caroll \& C. Von Stutterheim (2003) parlent dans ce cas de «Preferred patterns of information organisation ».

Les configurations discursives sont profondément enracinées dans le système grammatical de la langue source et constituent la trame fondamentale pour la production en langue étrangère ( $c f$. la notion de «thinking for speaking» de D. I. Slobin), qu'elles influent jusqu'à un niveau avancé. M. Carroll \& C. Von Stutterheim (2003) suggèrent même que les différences entre les variétés de L2 d'apprenants très avancés et la variété native résident précisément dans ces agencements préférentiels de l'information transmise. Cette hypothèse de type pragmatique peut également avoir un impact sur les options morphologiques ou syntaxiques choisies pour combiner et représenter des évènements.

L'influence de la L1 pour l'expression du mouvement en L2 a surtout été examiné pour l'acquisition de l'espagnol L2 et de l'anglais L2 (cf. à ce propos Cadierno, 2004, 2008 ; Cadierno \& Ruiz, 2006; Cifuentes Férez, 2009). L'objectif général de notre recherche est d'analyser les stratégies pour exprimer des évènements impliquant un mouvement en français L2, utilisées par des apprenants ayant pour L1 différentes langues romanes et germaniques (espagnol, allemand, anglais) et de les confronter à celles employées par des locuteurs de français L1.

\subsection{Les stratégies en français et en allemand $L 1$}

7 La présente contribution se limite à la comparaison de la production des apprenants germanophones à celle des locuteurs francophones natifs. Dans l'expression 
d'évènements marquant le mouvement, les deux langues s'opposeraient du point de vue typologique :

- par le recours préférentiel aux verbes de mouvement exprimant la manière de bouger (ou $\mathrm{M}$-verbes) en ce qui concerne l'allemand, ou aux verbes de mouvement exprimant la trajectoire (ou T-verbes) dans le cas du français ;

- par l'utilisation de satellites, de particules adverbales pour exprimer la trajectoire en allemand, contrairement au français.

$8 \quad$ L'opposition typologique entre les deux langues doit toutefois être nuancée. La tendance en français ne semble en effet pas toujours univoque. Ainsi, J. Kramer (1981) relève des interférences avec les options structurales des langues germaniques pour le français de Bruxelles (et l'explique par l'influence du néerlandais). En français standard, on attend des combinaisons du type : entrer, rentrer, descendre en sautant; mais à Bruxelles, on trouve des expressions qui ne sont pas courantes en français standard, par exemple: entrer dedans (< binnengaan); aller de retour (< teruggaan); sauter en bas (< naar beneden springen). Des exemples similaires ont été relevés en Alsace et en Lorraine (contact allemand/ français) et en Suisse (cf. l'étude de R. Berthele, 2004: 117 sur les langues en Suisse) : on y utilise à la place de descendre ou de monter les expressions aller aval ou venir amont, ce qui souligne que les frontières entre « manner languages » et «path languages» sont poreuses. Ces «interférences» tendraient cependant à diminuer lorsqu'on quitte les régions bilingues.

D'autre part, la faible exploitation de particules adverbales en français est également contestable. Celles-ci sont généralement analysées dans les grammaires comme des " prépositions à régime implicite » (Grevisse, Goosse, 1991 : § 992), ce qui leur ferait subir une « adverbialisaton » (Wilmet, 2010: §335, 343) ou une « réadverbialisation (Cervoni, 1991 : 105). La préposition, dite " orpheline ", qui subsiste telle quelle ou sous une forme modifiée en de (Riegel, 2009: 404), n'est autorisée en français standard qu'avec des prépositions « fortes » (Zribi-Hertz, 1984)1. Ces formes prépositionnelles « orphelines » ne se limitent pas à des emplois populaires comme l'affirme certains puristes (Hanse, 1987 : $86,318,331)$ :

- dedans et dehors: la particule s'utilise avec des verbes d'état ou de processus : être dedans/ dehors, mettre dedans/dehors (2a-b), mais la combinaison d'un M-verbe avec une particule marquant la destination (2c-d) n'est pas impossible. La récupération de l'argument peut se faire de manière cotextuelle ou contextuelle (2a). Dans certains cas on aura même affaire à un argument nul (2b) :

(2a) Je le croyais hors de la maison, il était dedans. (Grevisse)/ Ça ne rentre pas dedans. (Riegel)

(2b) Quelqu'un lui est rentré dedans. (Riegel)

(2c) Après quelques secondes, il court dehors pour alerter le voisinage. Il se force à pleurer pour donner le change. (Grouille, D., 2001, Horreurs médicales, manuscrit.com, p. 103)

(2d) Il a volé vers la grande fenêtre chez Line car il pensait s'envoler dehors. Seulement elle était fermée et il a foncé tout droit dans la vitre. (en ligne : chez.manon.free.fr/chezmanon5/pethist/44/44.htm, consulté le 04/06/13)

- dessus et dessous : ici aussi, les prédicats sont plutôt de type « état » ou « processus », mais la combinaison avec un verbe de mouvement (M-verbe + particule de direction/position, 3a-b) ne semble pas impossible et le caractère adverbal de la particule s'affirme (3c). L'argument sera récupérable de manière co- (3b) ou contextuelle (3a) et il pourra même être nul (3c) : 
(3a) Ecrire l'adresse dessus/ On lui a craché dessus. (Riegel)

(3b) Avec le football de rue, les meilleurs joueurs du monde sont toujours disponibles, mais le jeu prend une nouvelle dimension. Les terrains fermés ou les gymnases vous permettent d'utiliser les murs pour effectuer des passes surprenantes ou pour grimper dessus pour passer un défenseur adverse. (en ligne : http://www.nintendo.fr/Jeux/Wii/FIFA-11-281221.html, consulté le 04/06/13)

(3c) Elles viennent pourtant de ridiculiser Debrecen 43-22 en championnat de Hongrie ? «C'est encore plus excitant. Il va falloir leur grimper dessus", sinon elles vont nous manger tout cru ». (En ligne : http://www.republicainlorrain.fr/sports/2011/10/29/allison-pineau-on-va-leur-grimper-dessus, consulté le 04/06/13)

- devant, derrière et après : dans ces trois cas également, la combinaison avec un verbe de mouvement est autorisée (4a-d : M-verbe + particule marquant la position/trajectoire) :

(4a) L'un de nos proverbes assure que celui qui a une montre n'a pas le temps. Il court derrière sans jamais l'attraper. (Arzalier, F., 2002, Aujourd'hui l'Afrique 83, p. 30)

(4b) Les uns attendent les emplois, les autres courent après (Grevisse)

(4c) Arrête de lui courir après (Riegel)

(4c) Celui qui réussit, gagne sa place dans le monde comme ceux qui allaient autrefois au théâtre un soir de première : en jouant des coudes pour passer devant. (en ligne: http://www.rbc.com/aproposderbc/bulletin/ avril1949.html, consulté le 04/06/13)

(4d) Connexions : Walibi : payer plus pour passer devant, est-ce immoral ? (en ligne : http://www.rtbf.be/video/detail_connexions, consulté le 04/06/13)

L'ensemble des prépositions orphelines tendent donc à remplir une fonction assimilable à celle des particules adverbales de l'allemand. Comment alors l'opposition typologique entre l'allemand et le français dans l'utilisation de particules adverbales dans l'expression du mouvement se manifestera-t-elle sur le plan de l'appropriation du français L2 par des apprenants germanophones? Si la différence ne réside pas dans la présence ou l'absence de particules adverbales, l'impact de la L1 typologiquement distincte pourrait se manifester à travers certaines déviances dans l'emploi de ces dernières.

Plus spécifiquement, l'allemand utilise les satellites adverbaux pour marquer la trajectoire auprès d'un $\mathrm{M}$-verbe $(5 \mathrm{~b})$, tandis que le français utilise généralement un $\mathrm{T}$ verbe principal (5a) :

(5a) puis alors le cerf, il part en courant (5FFL1_053)

(5b) et le chien, il saute dehors (5DEFL2_02a)

Cette contribution examinera si la propension vers l'utilisation de M-verbes par les locuteurs germanophones a des conséquences sur une fréquence quantitative plus élevée et une utilisation qualitative différente des satellites adverbaux marquant la localisation (position, direction, trajectoire) dans la production de ces derniers. 


\section{Données recueillies et méthodologie}

13 L'étude de l'impact de la L1 sur la variation dans l'expression linguistique du mouvement en FL2, mais aussi de la variation existant dans la production des locuteurs natifs euxmêmes sera fondée sur une comparaison de deux corpus de locuteurs :

- un corpus de 30 apprenants germanophones de FL2 (dorénavant DEFL2), entre 10 et 12 ans, élèves des écoles européennes à Bruxelles ;

- un corpus de 30 locuteurs natifs (dorénavant FFFL1) du même âge (10-12 ans), également élèves dans les écoles européennes à Bruxelles .

14 Ceux-ci ont tous réalisé la même tâche orale narrative (narration de la "frog story", Mayer, 1969, cf. annexe 1). La quantité de texte produit par les deux groupes est hautement comparable :

- DEFL2 : N total mots : $17320^{4} ; \mathrm{N}$ moyen d'occurrences/ texte : 577,3 ;

- FFFL1 : N total de mots : 16986 ; N moyen d'occurrences/ texte :566,2.

La différence entre la longueur moyenne des deux séries de textes est donc de moins de $2 \%$, ce qui est négligeable.

Nos observations se donnent pour objectif de tester la pertinence d'une série d'hypothèses avancées dans la littérature traitant de cette problématique de recherche décrite sous le $§$ 1.3.3. (b). Elles se traduisent par les questions de recherche $(\mathrm{Q})$ suivantes :

Q1: Les particules adverbales marquant la trajectoire (la cible), sont-elles plus souvent convoquées par les apprenants DEFL2 que par le groupe FFFL1?

Q2 : Y a-t-il une différence dans l'emploi de ces particules entre les deux groupes?

\section{Fréquence et variation des particules adverbales de localisation}

Comme déjà mentionné dans 1.3.2, les particules adverbales du français marquant la trajectoire (la cible) sont les suivantes: dehors, dedans, derrière, devant, après, dessus ou dessous. Le tableau ci-dessous reprend l'ensemble des diverses particules adverbales susceptibles de marquer des aspects de la trajectoire/localisation, ainsi que la fréquence relative de chacune par rapport à l'ensemble.

Tableau 1. Fréquences absolues et relatives des particules adverbales chez les apprenants et les natifs

\begin{tabular}{|l|l|l|l|l|}
\hline PARTICULES & \multicolumn{2}{|l|}{ DEFL2 } & \multicolumn{2}{l|}{ FFFL1 } \\
\hline & $\mathrm{N}$ & $\%$ & $\mathrm{~N}$ & $\%$ \\
\hline dehors & 11 & 0,34 & 10 & 0,32 \\
\hline dedans & 14 & 0,44 & 3 & 0,10 \\
\hline derrière & 4 & 0,13 & 10 & 0,32 \\
\hline après & 0 & 0 & 5 & 0,16 \\
\hline
\end{tabular}




\begin{tabular}{|l|l|l|l|l|}
\hline devant & 1 & 0,03 & 1 & 0,035 \\
\hline dessus & 2 & 0,06 & 2 & 0,065 \\
\hline TOTAL & 32 & 1,00 & 31 & 1.00 \\
\hline
\end{tabular}

\subsection{Observations générales}

18 Sur le plan de la fréquence d'emploi globale des particules adverbales, les données révèlent peu de différence entre les groupes DEFL2 et FFFL1. La fréquence d'emploi des particules adverbales est donc similaire chez les natifs et les apprenants FL2 germanophones. Toutefois, la variation interne dans l'utilisation des diverses particules par les deux groupes révèle des disparités importantes :

a. la particules la plus fréquemment employée n'est pas la même dans les deux groupes : dedans (DEFL2), dehors, derrière (FFFL1) ;

b. dedans, très fréquent chez les apprenants DEFL2, est très peu utilisé par le groupe FFFL1;

c. après n'est pas utilisé par les apprenants germanophones comme particule adverbale ;

d. seule parmi les emplois significatifs, la particule dehors présente une fréquence d'emploi comparable dans les deux corpus.

\subsection{Emploi spécifique des particules de localisation}

\subsubsection{Dedans}

19 La particule est rarement utilisée dans le récit des natifs FL1. Elle apparait essentiellement avec des verbes d'état (5a) ou de processus (5b) pour marquer une localisation. Seul l'énoncé (5c) montre la combinaison d'un verbe partiellement $\mathrm{M}$ avec la particule orientant la trajectoire vers une cible :

(5a) Ils ont vu un tronc d'arbre et ils ont cru que la grenouille était dedans.

(5FFL1_04)

(5b) Et après, le bocal, enfin, il s'est mis la tête dedans. (5FFL1_08)

(5c) Et en dessous, heureusement qu'il y avait une rivière. Ils y sont tombés

dedans. (5FFL1_04)

Son emploi est nettement plus fréquent et varié dans la production des apprenants DEFL2. Tout d'abord, l'emploi dans le contexte de prédicats d'état ou de processus pour marquer la localisation est également productif :

(6a) là où (il) y a les abeilles qui sont dedans. (5DEFL2_08)

(6b) la uh@i grenouille \# dans l'arbre et \#\# il y a un hibou \#\# là+dedans.

(5DEFL2_02)

(6c) un truc [*] avec du l(a) eau dedans. (5DEFL2_05)

(6d) le garçon grimpe sur une arbre et \# (il) y a une trou dedans. (4DEFL2_01)

21 La combinaison d'un verbe (partiellement) $\mathrm{M}^{5}$ avec la particule orientant la trajectoire vers la cible est fréquente chez les apprenants DEFL2 :

(6e) et puis il a laissé tomber le chien et le garçon dans l'étang \# ils sont tombés dedans. (5DEFL2_08) 
(6f) truc l'eau <alors quand ils s@l> uh@i alors ils tombent là-dedans .

(5DEFL2_02a)

(6g) et dans le lac uh@i oui ils tombent dedans (DEFL2_01)

(6h) et le garçon uh@i (il) y a un trou au terre \# et uh@i il cherche là

+dedans. (5DEFL2_02)

(6m) là il y a des petits animaux \# qui vient uh@i uh@i là-dedans.

(5DEFL2_02)

(6n) uh@i ils vont \#\# partir plus dedans dans la forêt \#. (1DEFL2_03)

(6i) et il \# il crie le nom de la grenouille dedans (4DEFL2_01)

(6j) et $1 @ 1 ~[/ /]$ le garçon a encore vu un trou dans l'arbre et il a uh@i \# regardé dedans \# et (il) y a un hibou qui est sorti. (5DEFL2_08)

(6k) et puis il \# il veut regarder là-dedans. (5DEFL2_08)

(61) alors il regarde dedans et il voit \# uh@i sa grenouille avec une autre grenouille. (5DEFL2_08)

Plus surprenant est l'exploitation des particules auprès de T-verbes pour indiquer la cible

Enfin, les apprenants DEFL2 utilisent aussi dedans dans un contexte de locution prépositionnelle (dedans de), ce qui compense partiellement la fréquence moins élevée de l'emploi de dans chez les apprenants :

(6p) après il part uh@i plus dedans de la forêt \#. (1DEFL2_03)

\subsubsection{Dehors}

Non seulement la fréquence d'emploi, mais aussi les types d'emploi sont similaires dans la production des deux groupes. Voyons d'abord l'emploi avec des verbes d'état où la particule indique une localisation :

(7a) et la grenouille elle [/] \# elle \# veut être dehors (4DEFL2_01)

(7b) puis après, ils essaient de trouver dehors (4FFL1_04)

(7c) ils crient le nom de la grenouille qui est dehors. (5FFL1_01)

La combinaison d'un verbe (partiellement) $\mathrm{M}$ avec la particule orientant la trajectoire vers la cible est fréquente chez les apprenants DEFL2 :

(7d) et uh@i il saute dehors. (1DEFL2_03a)

(7e) et le chien, il saute dehors (5DEFL2_02a)

(7f) et le chien et lui ils cherchent dans toute la chambre et dehors

(1DEFL2_03)

(7g) Il cherche avec le chien ici dehors. (1DEFL2_03a) /

(7h) ils ont cherché partout et même dehors (5FFL1_04)

(7i) puis il cherche dehors. (5FFL1_06)

(7j) et il la cherche dehors et tout (5FFL1_02a)

La combinaison est aussi courante avec le verbe de mouvement prototypique aller, où la particule spécifie la trajectoire : 
(7k) et uh@i cherchent uh@i dans le [/] le forêt, \&oui uh@i va dehors

(5DEFL2_06)

(7l) Et dans la nuit, le grenouille va dehors (1DEFL2_01a)

(7m) Il (le garçon) va dehors (1DEFL2_01a)

(7n) Alors, il va dehors pour chercher le grenouille (1DEFL2_01a)

(7p) alors il s'habille pour aller dehors (5FFL1_08)

\section{contradiction avec le processus exprimé par le verbe de mouvement :}

(7v) et nuit la grenouille sort de le verre et passe par la fenêtre dehors

(5DEFL2_04a)

$(7 \mathrm{w})$ il y a son chien qui tombe dehors. (5FFL1_06)

\title{
3.2.3. Derrière et après
}
(8a) le chien tombe [/] tombe et le garçon regarde derrière lui. (4DEFL2_01)
(8b) les \# insectes vont derrière lui (4DEFL2_01)
(8c) et le hibou était encore derrière lui (4DEFL2_07)
(9a) le petit garçon il va sur un gros caillou il uh@i crie après le chien (1DEFL2_05a)
(9b) l'animau@il [:=il animal] il court uh@i après le chien et il s(e) arrête à [/ ] à [//] devant l'eau (1DEFL2_05a)
(9c) doucement il regarde après l'arbre. (1DEFL2_05a)

\begin{abstract}
Dans l'emploi de derrière en tant que particule, le M-verbe indique dans une grande majorité des cas un mouvement, localisé par la particule adverbale par rapport à une cible statique dans toutes les occurrences de DEFL2 et dans la plupart des occurrences de FFFL1. C'est uniquement cet emploi qui apparait dans la production du groupe DEFL2. Ce type d'énoncé permet souvent de sélectionner des modes de mouvement liés à divers organes humains (l'ouïe, le regard; cf. 10b-d et $11 \mathrm{c}-\mathrm{i}$ ) :
\end{abstract}

(10a) et uh@i il va derrière <et il prend> [//] uh@i le petit garçon uh@i avec le uh@i chien prend le grenouille (4DEFL2_03a)

(10b) ils ont \&hm cherché dans [//] derrière un arbre qui était déjà [/] uh@i 
déjà cassé et puis ils ont regardé derrière (4DEFL2_02a)

(10c) après il regardé uh@i uh@i derrière. (5DEFL2_06)

(10d) et après ils gardent uh@i derrière (4DEFL2_03a)

(11a) et il cherche derrière (4FFL1_07)

(11b) puis il y a un cerf qui l'attrape avec ses cornes derrière (5FFL1_06)

(11c) et il a regardé derrière (4FFL1_06)

(11d) et puis ensuite, il regarde derrière (1FFL1_02)

(11e) il regarda sans faire de bruit derrière (5FFL1_03)

(11f) et il regarde derrière très silencieusement (5FFL1_01)

(11g) il regarde derrière et voit Victor avec une femme grenouille (5FFL1_01)

(11h) après, ils ont regardé derrière (5FFL1_08)

(11i) et il entend des bruits derrière. (5FFL1_01)

Parfois avec derrière et toujours avec après chez les natifs FL1, la particule exprime la trajectoire du mouvement avec un M-verbe. Ce type d'emploi n'est jamais relevé dans la production des apprenants DEFL2, mais apparait régulièrement dans les récits des natifs :

(12a) et il y avait toutes les guêpes qui lui couraient derrière (1FFL1_05a)

(12b) (les abeilles) elles lui courent après (1FFL1_02)

(12c) et elles lui courent après. (1FFL1_07a)

(12d) et ici, il lui court après. (1FFL1_07a)

(12e) et le chien court après (5FFL1_06)

(12f) donc les abeilles lui courent après (5FFL1_02a)

\section{Observations concernant les particules adverbales de localisation}

De la description de l'utilisation des particules adverbales dans le récit par les deux groupes DEFL2 et FFFL1, ressortent diverses tendances permettant de caractériser l'exploitation de ces particules par les apprenants germanophones et par les natifs.

\subsection{Emploi des verbes de mouvement neutres}

Les apprenants du groupe DEFL2 utilisent de manière intensive les verbes de mouvement "neutres» (aller, être au sens de "se mouvoir») combinés avec une particule de localisation permettant de préciser la trajectoire :

(13a) Et dans la nuit, le grenouille va dehors (1DEFL2_01a)

(13b) Il (le garçon) va dehors (1DEFL2_01a)

(13c) Alors, il va dehors pour chercher le grenouille (1DEFL2_01a)

(13d) et uh@i cherchent uh@i dans le [/] le [*] forêt, \&oui uh@i va dehors.

(5DEFL2_06)

(13e) et uh@i il va derrière <et il prend> [//] uh@i le petit garçon uh@i avec le uh@i chien prend le grenouille (4DEFL2_03a)

(13f) et ici le garçon croit que là il y a un grand roche et il va dessus.

(1DEFL2_01a)

Les natifs du groupe FFFL1 utilisent rarement aller + particule de localisation, il y a une seule attestation dans le corpus :

(13g) alors il s'habille pour aller dehors (5FFL1_08) 
L'observation est confirmée par l'emploi fréquent de compléments de trajectoire après aller chez les apprenants germanophones, tandis que cette structure n'apparait pas chez les natifs :

(14a) et après les abeilles vont derrière le chien. (5DEFL2_01a)

(14b) les \# insectes vont derrière lui (4DEFL2_01)

(14c) et le hibou était encore derrière lui (4DEFL2_01)

(14d) et les abeilles ils [*] sont toujours derrière le chien. (5DEFL2_08)

(14e) un cerf le prend <sur le> [//] sur la tête \# et le chien uh@i \# va après

lui. (1DEFL2_03a)

(14f) le petit garçon, il va sur un gros caillou (1DEFL2_05a)

Cette stratégie spécifique des apprenants DEFL2 pour l'expression du mouvement peut s'expliquer par le besoin de pallier un lexique verbal encore défaillant. Ce choix est sans doute aussi favorisé par le caractère satellitaire (S-langue) de l'allemand L1.

\subsection{Collocation de particules de localisation et de certains verbes}

Un autre constat qui ressort des données observées est la tendance à la collocation de particules de localisation et de certains verbes, avec à nouveau des disparités entre la production des apprenants germanophones et des natifs :

Le M-verbe courir se combine de manière remarquable avec des particules, surtout dans la production des natifs L1 (seul 15a en production DEFL2) :

(15a) et le chien court devant. (4DEFL2_01)

(15b) et le chien courait devant (1FFL1_05a)

(15c) et il y avait toutes les guêpes qui lui couraient derrière (1FFL1_05a)

(15d) (les abeilles) elles lui courent après (1FFL1_02)

(15e) et puis, elles lui courent après. (1FFL1_07a)

(15f) et ici, il lui court après. (1FFL1_07a)

(15g) et le chien court après (09BRU2_5FRFR06)

(15h) donc les abeilles lui courent après (5FFL1_02a)

(15i) elles lui courent après, enfin elles volent après lui. (1FFL1_02)

Le M-verbe sauter et le T-verbe tomber (avec la possibilité d'interprétation de ce dernier en tant que M-verbe) sont souvent associés à des particules dans le discours des apprenants FL2, où l'aspect «manière » de ces verbes de mouvement est souligné. Ainsi, la combinaison sauter/tomber + complément d'orientation ne se retrouve jamais chez les natifs FL1, alors qu'elle est fréquente dans la production des apprenants germanophones ( cf. 17a-c) :

(16a) et dans le lac uh@i \&oui ils tombent dedans (1DEFL2_01)

(16b) et puis il a laissé tombé le chien et le garçon dans l'étang \# ils sont tombés dedans. (5DEFL2_08)

(16c) $[/]$ truc l'eau <alors quand ils s@l> [//] uh@i alors ils tombent làdedans. (5DEFL2_02a)

(16d) Et en dessous, heureusement qu'il y avait une rivière. Ils y sont tombés dedans. (5FFL1_04)

(16e) il y a son chien qui tombe dehors. (5FFL1_06)

(16f) et uh@i il saute dehors. (1DEFL2_03a)

(16g) et le chien, il saute dehors (5DEFL2_02a) 
(17a) et le chien avait un peu peur et sautait de la fenêtre en bas (4DEFL2_01)

(17b) il chutse en bas (5DEFL2_02)

(17c) et le chien tombe en bas par la fenêtre (1DEFL2_03) « un mouvement pour aller voir ». Ce type de collocation est largement exploité avec diverses particules aussi bien chez les natifs FL1 que chez les apprenants germanophones FL2 : peut être le fruit d'une stratégie d'appropriation provenant de l'allemand L1 (cf. il lui court après / il tombe dehors/ de jongen loopt het huis uit/ Der Junge rannte (aus dem Haus) hinaus), le nombre d'occurrences en FL1 pourrait aussi indiquer qu'il s'agit d'un développement par collocation de véritables satellites de localisation en français familier, voire standard.

\section{Conclusions}

De manière générale, les données de notre étude font ressortir que, sur le plan de l'exploitation des particules adverbales de trajectoire, l'opposition entre V-langues et Slangues s'impose de manière moins tranchée que ce que les analyses typologiques laissaient entendre. En résumé, l'hypothèse qui liait la prédilection pour des M-verbes dans le cadre d'une S-langue (à satellites) en L1 à l'utilisation plus fréquente de particules de trajectoire en L2 n'est pas vraiment confirmée dans la production du groupe DEFL2. Ce constat pourrait être lié :

a. au contexte et au processus d'apprentissage de la L2 ;

b. au fait que la distinction entre les types de langues soit moins tranchée que l'on ne affirme.

En effet, le contexte et le processus d'apprentissage sont assez particuliers. Les apprenants germanophones de FL2 sont des élèves des écoles européennes de Bruxelles. Dans l'école comme dans l'agglomération urbaine où ils habitent, le français est probablement la langue de communication dominante. Cela signifie que ces apprenants sont sans doute systématiquement confrontés aux configurations discursives 
particulières au français (les "preferred patterns of information organisation ») et que cela contrebalance quelque peu une possible influence de leur L1 sur la manière dont ils rendent compte d'évènements exprimant un mouvement.

Divers constats avaient déjà mis en évidence une large extension de l'emploi des particules de localisation en français oral, voire dans la langue standard (1.3.2). Ils sont corroborés par des études récentes qui tendent à nuancer et à moduler la théorie sur les verbes de mouvement et la distinction entre V- ou S-langues (cf. Ibarretexe-Antuñano, 2009) : un classement des langues sur la base de la prise en compte de la quantité et la qualité d'information sur la trajectoire fournie en dehors de la forme verbale situe le français (69\% d'information extérieure) entre l'espagnol (63\%) et l'allemand (74\%), soit exactement à mi-chemin entre celle qui est considérée plutôt comme un exemple de Vlangue (l'espagnol) et celle qui est plutôt vue comme une S-langue (l'allemand).

Ceci dit, certaines discordances apparaissent néanmoins dans l'utilisation des particules adverbales entre la production des apprenants germanophones et celle des natifs qui semblent indiquer une influence nuancée des oppositions postulées entre V-langues et Slangues. Si la quantité des particules convoquées est similaire dans le discours des deux groupes, la variété d'emploi et l'apparition de certaines « déviances » distinguent les deux productions :

- la production des apprenants germanophones se caractérise par un suremploi de la particule dedans et la quasi-absence de particules plus complexes, telles derrière ou après. Le groupe DEFL2 utilise également abondamment le verbe de mouvement «neutre » aller en combinaison avec une particule de trajectoire ;

- la production des natifs est plus équilibrée et variée en ce qui concerne l'emploi des diverses particules adverbales. Toutefois, l'emploi de dedans est fort rare, tout comme d'ailleurs l'emploi du verbe aller avec une particule de trajectoire.

Enfin, la collocation des particules avec certains verbes s'impose dans la production des deux groupes, mais pas exactement selon les mêmes modalités. C'est essentiellement dans ce contexte qu'apparaissent des formes « exclues».

\section{BIBLIOGRAPHIE}

BERTHELE, R. (2006). Ort und Weg. Die sprachliche Raumreferenz in Varietäten des Deutschen, Rätoromanischen und Französischen. Berlin: De Gruyter.

BERMAN, R. \& SLOBIN, D. I. (eds) (1994). Relating events in narrative: A crosslinguistic developmental study. Hillsdale, $\mathrm{NJ}$ : Erlbaum.

CADIERNO, T. (2004). «Expressing motion events in a second language : a cognitive typological research ». In: Achard, M. \& Neimeier, S. (eds), Cognitive linguistics, second language acquisition and foreign language pedagogy. Berlin : De Gruyter, p. 13-49.

- 2008). « Learning to talk about motion in a foreign language ». In: Robinson, M. P. \& Ellis, N. C. (eds), Handbook of cognitive linguistics and second language acquisition. London : Routledge,

p. 239-274. 
CADIERNO, T. \& RUIZ, L. (2006). « Motion events in second language acquisition ». Annual Review of Cognitive Linguistics 4, p. 183-216.

CARROLL, M. \& VON STUTTERHEIM, C. (2003). « Typology and information organisation : perspective taking and language-specific effects in the construal of events ». In: Giacalone Ramat, A. (ed.), Typology ans second language acquisition. Berlin : De Gruyter, p. 365-402.

CERVONI, J. (1991). La Préposition - Étude sémantique et pragmatique. Paris : Duculot.

CIFUENTES FÉREZ, P. (2009). «A crosslinguistic study on the semantics of motion verbs in English and Spanish ». In : A Crosslinguistic study on the semantics of motion verbs in English and Spanish. Munich : LINCOM.

DE LORENZO ROSSELLO, C. (2002). Les Relations temporo-aspectuelles dans le récit oral en français et en castillan, langues premières et langues étrangères. Étude transversale du stade ultime de l'acquisition d'une langue étrangère. Thèse de doctorat, Université Paris X Nanterre.

DEMAGNY, A.-C. \& PAPROCKA, U. (2004). « L'acquisition du lexique verbal et des connecteurs temporels dans les récits de fiction en français L1 et L2 ». Langages 155, p. 52-75.

GReVISSE, M. \& Goosse, A. (1991). Le Bon Usage. Éd. refondue par A. Goosse. Paris : Duculot. HANSE, J. (1987). Nouveau dictionnaire des difficultés du français moderne. $2^{\mathrm{e}}$ éd. Paris : Duculot. HICKMANN, M. (2003). Children's discourse: person, space and time across languages. Cambridge : Cambridge University Press.

IBARRETXE-ANTUÑANO, I. (2009). « Path salience in motion events ». In: Jiansheng, G. et al. (eds)s Crosslinguistic approaches to the psychology of language: Research in the tradition of Dan Isaac Slobin. New York : Psychology Press, p. 403-414.

KING, R. (2000). The lexical basis of Grammatical Borrowing. Amsterdam : John Benjamins.

KRAMER, J. (1981). « Die Übernahme der deutschen und der niederländischen Konstruktion Verb + Verbzusatz durch die Nachbarsprachen ». In: MEID, W. \& HELLER, K. (Hrsg.), Sprachkontakt als Ursache von Veränderungen der Sprach- und Bewusstseinsstruktur: Eine Sammlung von Studien zur sprachlichen Interferenz. Innsbruck: Institut für Sprachwissenschaft der Universität Innsbruck, p. 29-140.

LAMBERT, M. \& VON STUTTERHEIM, C. (2011). « La recherche en L2 et les principes sous-tendant les organisations textuelles ». In: Trévisol-Okamura, P. \& Komur-Thilloy, G. (eds), Parcours, acquisition et didactique des langues. Les termes d'un dialogue. Paris : Orizons, p. 261-276.

MAYER, M. (1969). Frog, Where Are You? New York : Dial.

MÉLIS, L. (2003). La Préposition en français. Paris : Ophrys.

RIEGEL, M., PELLAT, J.-C. \& Rioul, R. (2009). Grammaire méthodique du français. $7^{\mathrm{e}}$ éd. revue et augmentée. Paris : Presses universitaires de France.

SLOBIN, D. I. (1996). « Two ways to travel : Verbs of motion in English and Spanish ». In:

Thompson, S. A. \& Shibatani, M. (Hrsg.), Grammatical constructions : Their formand meaning. Oxford: Oxford University Press, p. 195-217.

Slobin, D. I. (2004). «The many ways to search for a frog: Linguistic typology and the expression of motion events ». In: Strömqvist, S., Verhoven, L. (Hrsg.), Typological and contextual perspectives. Mahwah, NJ : Lawrence Erlbaum, p. 219-257.

- (2006): « What makes manner of motion salient ? Explorations in linguistic typology, discourse 
and cognition ». In: Hickmann, M. (Hrsg.), Space in languages. Linguistic systems and cognitive categories. Amsterdam : Benjamins, p. 59-81.

TALMY, L. (1985). « Lexicalization patterns : Semantic structure in lexical forms ». In: Shopen, T. (Hrsg.), Language typology and syntactic description. Cambridge : Cambridge University Press, p. 75-149.

- (2000). Towards a cognitive semantics. Cambridge, MA : MIT Press.

WiLMET, M. (2010). Grammaire critique du français. $5^{\mathrm{e}}$ éd. entièrement revue. Bruxelles : Duculot. ZRIBI-HERTZ, A. (1984). « Prépositions orphelines et pronoms nuls ». Recherches linguistiques de Vincennes, 12, p. 46-91.

\section{NOTES}

1. Certaines variétés nord-américaines, telle l'acadien, présentent aussi des énoncés avec des prépositions « faibles » (cf. King, 2000):

(a) Marie est une jeune fille que j'ai confiance en.

(b) Le gars que j'ai parlé à.

2. « Grimper dessus » signifie ici « se montrer dominant ».

3. Ce code permet d'identifier l'apprenant du corpus: le premier chiffre correspond à l'année d'enseignement et le dernier identifie l'apprenant au sein de la classe. Le code «FFL1 » renvoie aux apprenants natifs et le code «DEFL2 » renvoie aux apprenants germanophones de français L2.

4. Nombre brut: 20376 , dont il faut retirer les hésitations, les énoncés inaboutis, les interventions de l'intervieweur, soit environ $15 \%$ de la production globale.

5. Un verbe comme tomber peut être interprété comme un T-verbe (trajectoire du haut vers le bas), mais aussi comme un $\mathrm{M}$-verbe (manière de se mouvoir).

\section{RÉSUMÉS}

Les études typologiques sur l'expression du mouvement ont souligné une différence radicale entre les langues romanes et germaniques dans la réalisation linguistique de tels énoncés (Talmy 1985, 2000 ; Berman \& Slobin 1994). Les langues romanes préfèrent en général les verbes de mouvement qui marquent une direction ou une trajectoire («path»; par exemple en français sortir, descendre, monter ou en espagnol salir, bajar, montar), alors que les langues germaniques favorisent les verbes de mouvement qui expriment la manière de bouger («manner»; par exemple en anglais run, roll, creep, en néerlandais rennen, lopen, springen ou en allemand rennen, eilen, schleichen). De plus, les langues romanes sont des V-langues (centrées sur le verbe) dans la mesure où c'est celui-ci qui exprime l'orientation du mouvement. En revanche, les langues germaniques sont des S-langues (centrées sur le satellite) dans le sens où la trajectoire y est souvent indiquée par une particule adverbale (donc ajoutée au verbe) comme (en anglais in, out, up, en néerlandais in, uit, buiten, binnen, boven ou en allemand hinaus, hinein, hinauf).

Cette première étude exploratoire vise à évaluer l'impact du contraste invoqué sur la production, par des apprenants germanophones de français L2, de particules adverbales de trajectoire 
« exclues » en français L1, ainsi qu'à relever une éventuelle variation existant dans la production des locuteurs natifs eux-mêmes. Les données examinées font ressortir que, sur le plan de l'exploitation des particules adverbales de trajectoire, l'opposition entre V-langues et S-langues s'impose de manière moins tranchée que ce que les analyses typologiques laissaient entendre. Si la quantité des particules convoquées est similaire dans le discours des deux groupes, la variété d'emploi et l'apparition de certaines «déviances » distinguent les deux productions. Enfin, la collocation des particules avec certains verbes s'impose dans la production des deux groupes, mais pas exactement selon les mêmes modalités.

Typological studies on the linguistic expression of movement have emphasised a radical difference between Romance and Germanic languages (Talmy 1985, 2000 ; Berman \& Slobin 1994). Romance languages generally prefer movement verbs marking a direction or a trajectory (path: e.g. French sortir, descendre, monter or Spanish salir, bajar, montar), while Germanic languages favour movement verbs which express the manner of the movement (e.g. English run, roll, creep, Dutch rennen, lopen, springen or German rennen, eilen, schleichen). Moreover, Romance languages are V-languages (i.e. centred around the verb) because the verb itself expresses the orientation of the movement, whereas Germanic languages are S-languages (i.e. centred around the satellite) since the trajectory is often marked by an adverbial particle, which is added to the verb (English in, out, up, Dutch in, uit, buiten, binnen, boven or German hinaus, hinein, hinauf).

This first exploratory study aims to evaluate the impact of the mentioned contrast on Germanspeaking L2 French learners' production of adverbial particles marking the trajectory, which are "rejected" in L1 French, and to reveal the possible existing variation in the productions of French native speakers. The analysed data highlight that the opposition between V-languages and Slanguages, regarding the use of adverbial particles marking the trajectory, is not as sharply contrasted as the typological studies have suggested. The quantity of used adverbial particles is comparable in the productions of both groups, but the variety and the appearance of certain deviancies distinguish the two productions. Finally, the collocation of adverbial particles with certain verbs is noticed in the productions of both groups, but according the exact same modalities.

\section{INDEX}

Mots-clés : mouvement, typologie, acquisition, français L1, français L2, étude contrastive

Keywords : movement, typology, acquisition, French L1, French L2, contrastive study

\section{AUTEURS}

\section{MARIE-EVE MICHOT}

Talk, Frans, Vrije Universiteit Brussel

\section{STEFANIE GOLDSCHMITT}

Universität Würzburg

\section{MICHEL PIERRARD}

Talk, Clin, Frans, Vrije Universiteit Brussel, Belgique 